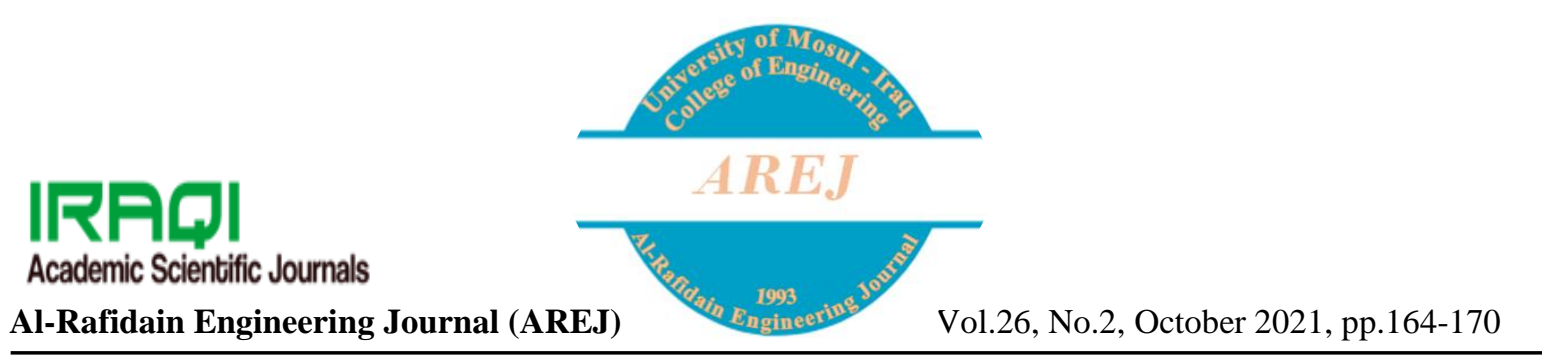

\title{
Study of the Impact of Antenna Selection Algorithms of Massive MIMO on Capacity and Energy Efficiency In 5G Communication Systems
}

\author{
Sawsan N. Abdullah \\ Farhad E. Mahmood \\ sawsan.enp8@student.uomosul.edu.iq \\ farhad.m@uomosul.edu.iq
}

Y. E. Mohammed Ali

a.yessar@uomosul.edu.iq

Electrical Engineering Department, Collage of Engineering, University of Mosul

Received: $12 / 6 / 2021$

Accepted: 24/7/2021

\begin{abstract}
Massive MIMO system in the fifth generation can consume a large amount of energy. In this research, the selection of antennas was studied based on several algorithms and a new algorithm was proposed for the selection of antennas. The results showed through a comparison between the approved and proposed methods in the research in terms of capacitance that The proposed algorithm is the closest in terms of capacity to the ideal case, as this method is considered almost ideal for its application in multi-input and multi-output systems to improve performance in the fifth generation, followed by the greedy algorithm and algorithm norm, as it was noted from During the comparison in terms of energy efficiency, it is possible to increase energy efficiency when choosing a certain number of antennas
\end{abstract}

Keywords:

Antenna Selection, Massive MIMO, 5G

This is an open access article under the CC BY 4.0 license (http://creativecommons.org/licenses/by/4.0/). https://rengj.mosuljournals.com

\section{INTRODUCTION (10 PT)}

Due to the rapid technical development in the previous wireless communication generations, the number of users are expected to increase tin times in the coming wireless generation of communication [1]. Furthermore, mobile traffic, in general, will increase by eight times. Figure.1 shows the data and the growth of traffic from 2016-2022. It is expected in 2022 that more than $90 \%$ of the traffic will be mobile phones [2], which calls for high data rates, high spectral efficiency, high energy efficiency [1], and larger network capacity [3]. The technology of (MIMO) is invented to meet the unprecedent demands of high data rate. In 5G (massive MIMO) technology is the extension of the traditional MIMO technology that involves the use of hundreds or even thousands of antennas connected to the base station to improve spectral efficiency and throughput. The increase in capacity, throughput, and spectral efficiency in large MIMO systems has made it a critical issue and has made it a critical technology for emerging wireless standards [2]. The deployment of a large number of antennas leads to an increase the energy consumption. Where,

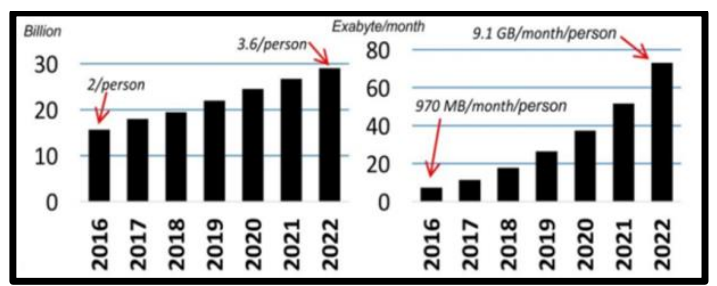


Figure 1 Global Mobile Data Traffic and Growth in Connected Devices from [2]

increasing the antenna push to increase the number of devices associated with antennas, including amplifiers (PA), digital to analog converters (ADC), analog to digital converters (DAC), (mixers), in transmitting and receiving antennas. Choosing a subset of antennas can be a solution to reduce the consumed energy. The basic idea of choosing an antenna with the highest channel gain. This may require the knowledge of the channel and some signal processing technique is available in the recent advanced massive MIMO system [6]. Choosing the optimum subset could be a challenging task as it added complexity to the process [4]. Many research articles have been presented in this field. The year of 2015 witnessed a new proposal, which is a binary switching architecture, as researchers, Xiang Gao, et al., proposed this method of selecting the antenna and it is considered the simplest solution and it is below the optimum level. However, it provides better signal quality. Where the proposed method was evaluated by comparing the total capacitance when using many binary switching and the results obtained through the proposed proposal that through the use of the proposed binary switch, the complexity is less and the loss in signal quality is less, but correspondingly the number of frequency chains (RF) is complete, i.e. without reducing the (RF) [5]. Fig 1 shows the data traffic and growth from 2016 to 2022 [2]. In 2017, researchers Yuan Gao, et al., presented a proposal for selecting antennas in large MIMO systems, which is the existence of two switching architectures, one is for the full channel array and the other is for the sub-matrix or for the channels that are selected. The assumed channel is Rayleigh flat fading channels. The results of the proposed algorithm showed that it could be applied when multiple- input and multioutput finite-dimensional MIMO systems were finite-dimensional [6]. In the paper of [7] a new algorithm will be proposed for selecting antennas and comparing them with the ideal case in systems of multiple inputs and huge outputs in terms of capacity, energy efficiency, and complexity.

In this paper, we study the performance of different antenna selection algorithms, and we proposed an algorithm that can provide the highest throughput at low processing time. Two algorithms have been investigated: the norm bases algorithm. The proposed algorithm then has been modeled and compared with the other mentioned algorithms.

This paper is organized as following sections: in Section two, we discuss the system model. In Section three, we explain different algorithm to select antenna that we use in the paper. The fourth section Energy Efficiency, results, and then a summary will be presented

\section{SYSTEM MODEL}

In the paper, The system used is single-user Massive MIMO(SU- MMIMO) we have antennas (Nt) at the base station and a receiving antenna $(\mathrm{Nr})$ at the user. From the Nt antennas, we will choose a subgroup antenna (Ns). The antenna selection process can be seen in Figure 2 . In the downlink scenario, data is transmitted from the base station over the Rayleigh channel. Since the number of receiving station antennas is much less than the base station, (Time Division Duple) TDD technology was used, which is one of the techniques in which the same frequency in transmitting and receiving data periodically, the downlink and uplink directions are separated in the time domain because the same frequency band is used and that $(\mathrm{H})$ represents the channel parameter and the received signal can be modeled as follows:

$y=H x+n$

Whereas:

$\mathrm{y}$ : Represents the transmitted signal

$\mathrm{x}$ : Represents the received signal

$\mathrm{n}$ : Represent noise

$\mathrm{H}$ : It represents the product of the number of transmitting and receiving antennas

where the channel is

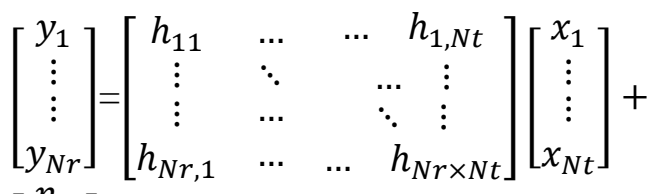

$\left[\begin{array}{c}n_{1} \\ \vdots \\ \vdots \\ n_{N r}\end{array}\right]$ 


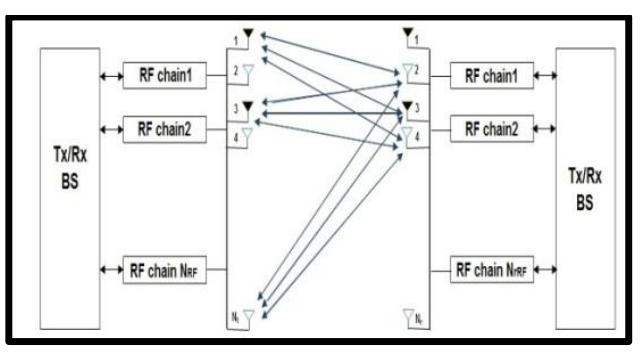

The figure2: shows the block diagram of the antenna selection process MIMO systems

Where the black pointer antenna indicates the selected active antennas, Otherwise, it refers to the antennas that have not been selected

\section{ANTENNA SELECTION}

In this section, the methods of selecting the antenna and comparing it with the proposed method will be explained. The received signal equation will be:

$y=H s \quad x+n$

Where Hs is the part of the total channel that we have the selected antenna.

\subsection{THE PROPOSED ALGORITHM}

A new method has been proposed for selecting antennas in MIMO wireless systems, which is a method inspired by the Multiple Signal Classification abbreviated (Music), which is the most common technique in practical applications due to the presence of an unknown number of signals, which makes up the channel array. The analysis of antenna selection is based on the analysis of the Eigen value decomposition of the channel matrix, by identifying the antennas that are located in front of the smallest value of the channel in the matrix and working on deactivating it, choosing the antennas with the highest channel value and activating them. In this method it is assumed that the full channel matrix $(\mathrm{H})$ is well known which is heterogeneous and is applied:

First: Eigen value decomposition of the component channel in order to convert the channel formed from a heterogeneous matrix to a homogeneous matrix.

Second: The Eigen value of the channel matrix, which represents the diagonal of the homogeneous channel matrix, is calculated.
Third: The orthogonal directions of the channel are determined by calculating the vector values (Eigen vector), and then the columns that contain the highest value of the channel are determined, which is the $(\mathrm{Hs})$ consisting of a subset of the active channels, and thus the subinformation in these columns contributes In increasing the capacity, this method is considered a type of digital signal processing, and this can be illustrated through the following equations:

$R^{d l}=\mid \log _{2} \operatorname{det}\left(I_{N r}+\frac{\gamma d l}{N r} H H^{H}\right)$

$H^{H}=V D V^{H}$

D: eigenvalues of channel matrix $\mathrm{H}$

$Z=\operatorname{argmax}\left[H^{H} V^{-1} H\right]$

where $\mathrm{V}$ is Eigenvector of channel matrix $\mathrm{H}$. The cardinality of $\mathrm{Z}$ is the number of selected antennas required. Then we can set $H_{S}=H(Z)$

$R_{S}^{d l}=\log _{2} \operatorname{det}\left(I_{N r}+\frac{\gamma d l}{N r} H_{S}^{H} H_{S}\right)$

This method gives a near-perfect comparison with the ideal algorithm, which has a lower computational complexity and reduces the actual time for selecting antennas, although it is a complex method in terms of numerical or numerical calculations, but it is suitable for a large number of antennas and is easy to apply in terms of practical performance compared to the ideal algorithm.

\subsection{GREEDY ALGORITHM ANTENNA SELECTION:}

In this algorithm where the antennas are sorted based on the nature of their channels in descending order, and in each iteration, the antenna with the highest channel will be selected, meaning that the mathematical analysis of this algorithm starts from calculating the highest standard for the channel and then these channels will be examined in terms of amplitude with the total rate if the More than the total capacity, the channel will be turned off, otherwise the antenna will be activated [8]. The difference between the norm algorithm and greedy algorithm is that in the first the antenna will be selected based on the highest channel 
standard, but in this algorithm, the antenna is selected after calculating the highest norm and examining all the columns that The criterion was calculated for it and the column with the highest capacity value was selected compared to the total capacity. It is considered an algorithm derived from the norm algorithm

\subsection{NORM ALGORITHM ANTENNA SELECTION (NBS):}

Since the channel is equal, where the implementation of any multi-input and output system requires a large number of frequency chains (RF), which is equal to the existing antennas. In practice, the number of chains is often limited and prices are limited, and, therefore, the number of antennas selected at the base station must be less than the number of antennas at the base station itself. The number of antennas selected at the base station is denoted by $(\mathrm{Ns})$, meaning that $(\mathrm{Ns}<\mathrm{Nt})$ and the number of antennas selected at the receiving station $(\mathrm{Lr})$ or (mobile) meaning that $(\mathrm{Lr}<\mathrm{Nr})$ and the full channel matrix $\mathrm{H} \in^{(\mathrm{CLr} \times \mathrm{Ns}))}$ is complex Gaussian values with zero mean and unit variance and size of $(\mathrm{Nr} \times \mathrm{Nt}))$. $\mathrm{H}$ is a (default) matrix since communication occurs only through the sub-channel matrix consisting of $(\mathrm{Lr} \times \mathrm{Ns})$ element. Where the symbols are sent through the active sub-channel matrix (H_S $\epsilon^{(\mathrm{CLr} \times \mathrm{Ns}))}$ where (H_S) is a subset of the $(\mathrm{H})$ channel matrix, and in general, increasing the channel gain is equivalent to reducing noise effects. This leads to improved system performance, and motivates us to apply the (NBS) algorithm, where the (H_S) finite relative to the. Matrix is selected The subchannel that has the highest standard and is part of the full channel matrix, where the relationship can be given through the following equation [7].

$$
\begin{aligned}
& R^{d l}=\log _{2} \operatorname{det}\left(I_{N r}+\frac{\gamma d l}{N r} H H^{H}\right) \\
& H_{s}= \\
& \operatorname{argmax}_{H s C H}\left\|H_{S}\right\|^{2}= \\
& \quad \operatorname{argmax}_{H s C H} \sum_{N_{t}=1}^{N s} \sum_{N_{r}=1}^{L_{R}}\left|H_{s}\left\langle N_{r}, N_{t}\right\rangle\right|^{2}
\end{aligned}
$$

MIMO systems, MIMO systems can achieve large gains in system power in two main ways, both of which depend on an increase in the size of the system. First: Increase in the number of antennas in the base station after what was its maximum in the fourth generation is eight, secondly, by increasing the number of antennas in the reception, it can achieve gains even at low levels of power. In the fifth generation that uses large multi-input and multi-output systems.

The energy efficiency equation can be given by the following equation[10]

$$
\begin{aligned}
& \text { energy efficiency }=\text { Capacity } / \text { total } \\
& \text { power....(11) }
\end{aligned}
$$

\section{RESULTS}

In this section, the performance of the proposed algorithm will be compared with the performance of the optimization algorithm. As this was done through the use of the Matlab program, the total number of antennas in the base station was set to $\mathrm{Nt}=128$ antennas, and the mobile station by $\mathrm{Nr}=2$ antennas, and $\mathrm{Ns}=$ 50 antennas were selected in the base station, respectively, and comparisons were made for the algorithms in terms of capacity, energy efficiency and complexity.

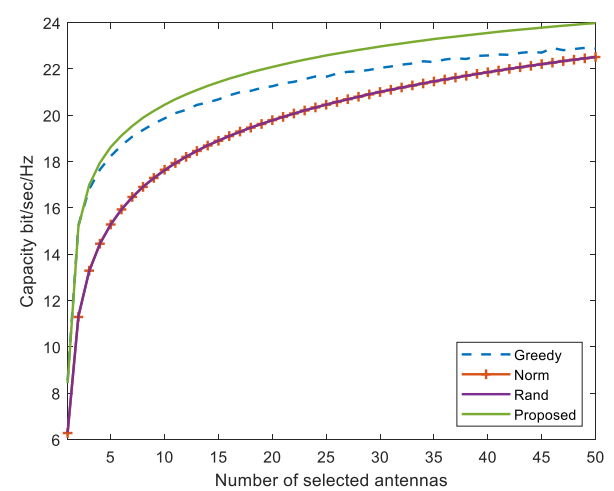

Figure.3: Capacity vs the number of the selected antenna $\mathrm{Nt}=128, \mathrm{Nr}=2, \mathrm{SNR}=20 \mathrm{~dB}$, $\mathrm{Ns}=50$
$R_{S}^{d l}=\log _{2} \operatorname{det}\left(I_{N r}+\frac{\gamma d l}{N r} H_{S} H_{S}^{H}\right)$

\section{ENERGY EFFICIENCY}

When comparing MIMO systems with traditional MIMO systems with multi-user 


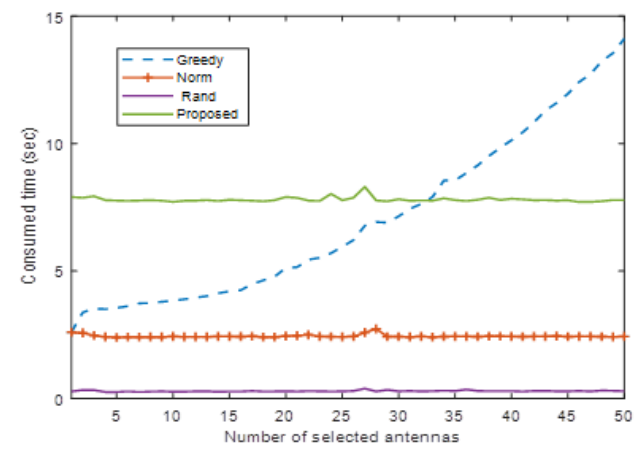

Figure.4: Time consumed as a function of number of selected antenna at $\mathrm{Nt}=128$.

$\mathrm{Nr}=2, \mathrm{SNR}=20 \mathrm{~dB}, \mathrm{Ns}=50$. Figure (3) and (4) show the relationship of the number of selected antennas with capacity and complexity. We note when choosing 50 antennas from among 128 antennas in the base station at $\mathrm{SNR}=20 \mathrm{~dB}$, it was found when changing or increasing the number of selected antennas and keeping the number of antennas fixed at the base station with a value of 128 antennas There is an increase in the amplitude of up to 23.98 [bits $/ \mathrm{sec} / \mathrm{Hz}]$ in the proposed algorithm and the complexity (time period) decreases with the increase in the selection of antennas which is $(7.777 \mathrm{sec})$, while the (Greedy) algorithm although the amplitude increased22.88 [ bits/sec/Hz] With the increase in antenna selection, the complexity or (time period) for antenna selection has increased to reach the limit of $(14.116 \mathrm{sec})$. 22.52[ $\mathrm{bits} / \mathrm{sec} / \mathrm{Hz}]$, but there is a difference in complexity (time period) because the difference in complexity between them is $(0.2847 \mathrm{sec})$ and $(2.4291 \mathrm{sec})$ respectively for randomness and norm. The difference between the algorithms can be seen through the following table 1

Table 1. shows the comparison between the algorithms

\begin{tabular}{|c|c|c|c|c|}
\hline & $\begin{array}{l}\text { The } \\
\text { Prop } \\
\text { osed } \\
\text { Algo } \\
\text { rith } \\
m\end{array}$ & $\begin{array}{l}\text { Greedy } \\
\text { Algorithm } \\
\text { Antenna } \\
\text { Selection }\end{array}$ & $\begin{array}{l}\text { Norm } \\
\text { Algorithm } \\
\text { Antenna } \\
\text { Selection } \\
\text { (NBS) }\end{array}$ & $\begin{array}{l}\text { Rando } \\
\mathrm{m} \\
\text { Algorith } \\
\mathrm{m} \\
\text { Antenn } \\
\mathrm{a} \\
\text { Selectio } \\
\mathrm{n}\end{array}$ \\
\hline $\begin{array}{l}\text { Capacity[bits/s } \\
\text { ec/Hz }\end{array}$ & 23.98 & 22.88 & 22.52 & 22.52 \\
\hline $\begin{array}{l}\text { Time } \\
\text { consumed } \\
\text { [sec] }\end{array}$ & 7.777 & 14.116 & 2.4291 & 0.2847 \\
\hline
\end{tabular}

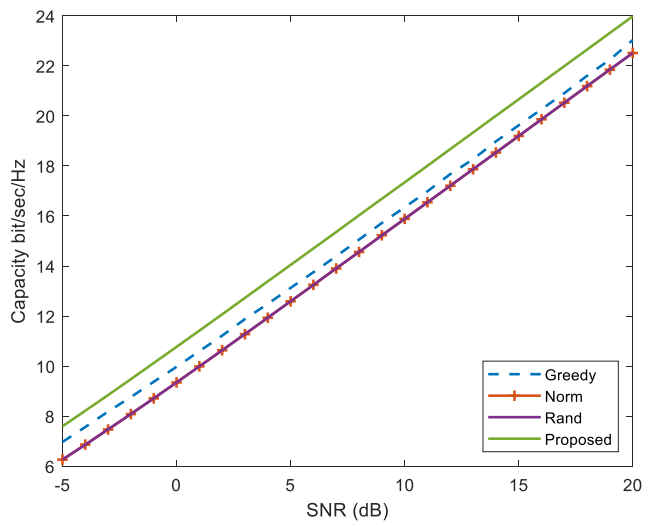

Figure.5 Capacity vs SNR Nt $=128, \mathrm{Nr}=2$, $\mathrm{SNR}=-5$ to $20 \mathrm{~dB}, \mathrm{Ns}=50$

The figure5 shows the relationship of the number of selected antennas versus the capacity when changing the SNR value. We note that the increase in the SNR value has a significant effect on increasing the capacity of the system, as we note that the selection of a fixed number of antennas and an increase in SNR, we note that there is an increase in the capacity

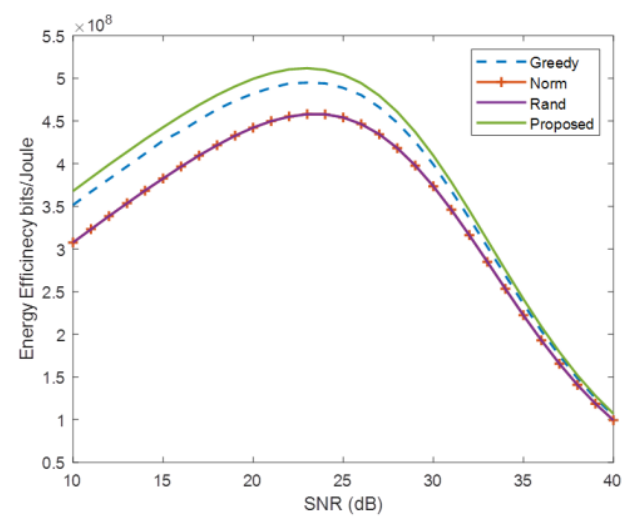

Figure.6 The Energy Efficiency bits/Joule vs. $\mathrm{SNR} N \mathrm{Nt}=128, \mathrm{Nr}=2, \mathrm{SNR}=10$ to $40 \mathrm{~dB}, \mathrm{Ns}=16$.

From the figure 6 , we notice that with the increase in antenna selection, the energy efficiency decreases. This can be explained when the number of selected antennas is few compared to the number of antennas in the base station, the number of (RF) is less, and thus the power consumption of the frequency chain (RF) is low compared to the transmitted power But when the number of selected antennas is close to the number of antennas of the base station or $(\mathrm{RF})$, the frequency chains increase, the power consumption of the frequency chain (RF) to the total energy will decrease and thus reduce the efficiency of the system, and thus the system that depends on the selection of the antenna 
achieves better energy efficiency From the old system without choosing the antenna or close to the (full selection), which proved that the proposed system is effective in using energy and enhancing performance in MIMO systems.

\section{CONCLUSION}

In this paper, we study the antenna selection algorithms to choose the best set of antennas out of a large number of transmit antennas to save energy. Different antenna selection algorithms have been under investigation. We consider the capacity, the energy efficiency, and the consumed processing time as a measure of the performance of these algorithms. Other algorithms provide different performances. We, also, proposed an algorithm to select the best set of antennas that provide a capacity close to that of optimum and with a much lower process time.

\section{Acknowledgements}

We thank the University of Mosul for providing us with facilities to complete this research

\section{References:}

[1] M. A. Albreem, M. Juntti, and S. Shahabuddin, -Massive MIMO Detection Techniques: A Survey,\| IEEE Commun. Surv. Tutorials, vol. 21, no. 4, pp. 3109-3132, 2019, doi: 10.1109/COMST.2019.2935810.

[2] R. Chataut and R. Akl, -Massive MIMO systems for $5 \mathrm{G}$ and beyond networks-overview, recent trends, challenges, and future research direction,\| Sensors (Switzerland), vol. 20, no. 10, pp. 1-35, 2020, doi: 10.3390/s20102753.

[3] M. Agiwal, A. Roy, and N. Saxena, -Next generation 5G wireless networks: A comprehensive survey,\| IEEE Commun. Surv. Tutorials, vol. 18, no. 3, pp. 1617-1655, 2016, doi: 10.1109/COMST.2016.2532458.

[4] S. Khalid, R. Mehmood, W. bin Abbas, F. Khalid, and M. Naeem, -Probabilistic distribution learning algorithm based transmit antenna selection and precoding for millimeter wave massive MIMO systems,\| Telecommun. Syst., vol. 76, no. 3, pp. 449-460, 2021, doi: 10.1007/s11235-020-00728-z.
[5] X. Gao, O. Edfors, F. Tufvesson, and E. G. Larsson, - Multi-Switch for Antenna Selection in Massive MIMO,\| pp. 1-6, 2016, doi: 10.1109/glocom.2015.7417765.

[6] Y. Gao, H. Vinck, and T. Kaiser, -Massive MIMO Antenna Selection: Switching Architectures, Capacity Bounds, and Optimal Antenna Selection Algorithms, IEEE Trans. Signal Process., vol. 66, no. 5, pp. 1346-1360, 2018, doi: 10.1109/TSP.2017.2786220.

[7] T. Cheng, Y. He, L. Shi, Y. Wu, Y. Huang, and Y. Sui, -A novel adaptive hybrid truncation precoding strategy in massive MIMO,॥ IEEE Commun. Lett., vol. 22, no. 11, pp. 2298-2301, 2018, doi: 10.1109/LCOMM.2018.2869153.

[8] A. Gorokhov, D. A. Gore, and A. J. Paulraj, - Receive antenna selection for MIMO spatial multiplexing: Theory and algorithms,\| IEEE Trans. Signal Process., vol. 51, no. 11, pp. 27962807, 2003, doi: 10.1109/TSP.2003.818204.

[9] R. Hamdi, E. Driouch, and W. Ajib, - Resource allocation in downlink large-scale MIMO systems, ॥ IEEE Access, vol. 4, pp. 8303-8316, 2016, doi: 10.1109/ACCESS.2016.2630999.

[10] K. N. R. S. V. Prasad, -E nergy E fficiency in M assive MIMO-B ased 5G N etworks: $\mathrm{O}$ pportunities and $\mathrm{C}$ hallenges,\| IEEE Wirel. Commun., pp. 2-10, 2016. 


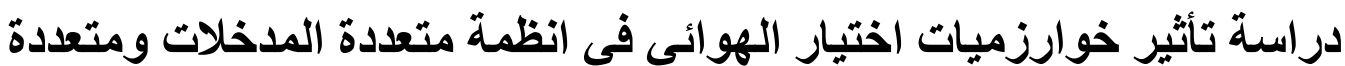

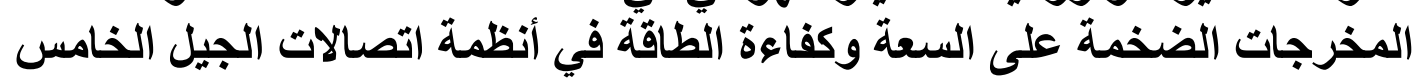

يسار عز الاين محمد علي

a.yessar@uomosul.edu.iq
فرهاد عزالدين محمود

farhad.m@uomosul.edu.iq sawsan.enp8@student.uomosul.edu.iq

جامعة الموصل ـ كلية الهندسة ـ قسم الهندسة الكهربائية

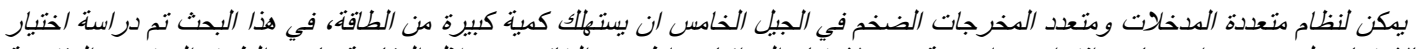

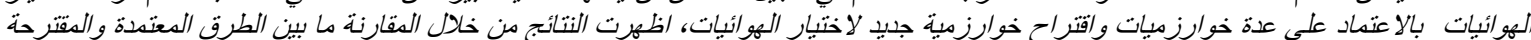

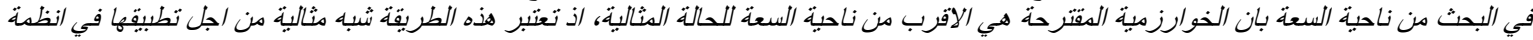

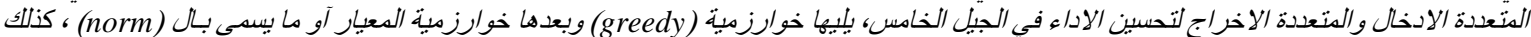
لوحظ من خلال المقارنة من ناحية كفاءة الطاقة انه بيكن الزيادة في كفاءة الطاقة عند اختيار عدد معين من الهوائيات

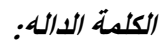

اختيار الهوائي، انظمة متعددة الددخلات ومتعددة المخرجات، الجيل الخامس 